\title{
La figura del Farmacéutico de Atención Primaria, nuevos retos
}

\begin{abstract}
C Cuando me pidieron que escribiera un editorial sobre las funciones y el trabajo que los farmacéuticos desarrollan en las estructuras de atención primaria (AP), me pareció una oportunidad importante para reflexionar en voz alta sobre nuestro pasado y, sobre todo, sobre nuestro futuro, haciendo partícipe de esa reflexión al colectivo de profesionales que han sido nuestros compañeros de viaje durante estos años.
\end{abstract}

Es indudable que la labor del farmacéutico de atención primaria (FAP) está consolidada, y actualmente totalmente respaldada por la ley. Esto, trasladado a nuestro ámbito de trabajo, se traduce en que los FAP llevan más de veinte años desarrollando gran variedad de funciones, la mayoría de ellas destinadas a diseñar estrategias y actividades de promoción del uso racional de medicamentos, mediante la evaluación crítica de la aportación terapéutica de los medicamentos, el trabajo con el valor económico de los mismos y, lo más difícil, la contribución a la difusión de todo ese conocimiento con veracidad y profesionalidad, y orientado a ayudar en la toma de decisiones en materia de medicamentos en AP. Creo, sinceramente, que esa definición, quizás un poco excesiva, de "expertos en el medicamento" se ha ganado a pulso, con una formación profunda sobre el conocimiento de la metodología para la evaluación de medicamentos, reconocido por la mayoría de los profesionales sanitarios que trabajan en las estructuras de la AP.

Recientemente se ha publicado una encuesta promovida por la Sociedad Española de Farmacéuticos de AP (SEFAP) y realizada entre los servicios de farmacia de AP de todo el territorio nacional. Los resultados de la misma confirman que las actividades destinadas a la promoción del uso racional de los medicamentos alcanzan porcentajes de desarrollo muy elevados.

Pero igual que hay luces, también hay sombras, ya que en la encuesta se pone de manifiesto que la vertiente clínica del FAP, teniendo al paciente como objetivo común (seguimiento, cumplimiento y continuidad farmacoterapéutica, seguridad y farmacovigilancia, educación sanitaria), es la menos desarrollada. Sólo el 10\% de los servicios de farmacia de AP realizan seguimiento de los pacientes, y solo el $27 \%$ tiene acceso a la historia clínica para realizar un correcto seguimiento. Quizás la excesiva institucionalización de nuestro colectivo hace que a veces sea la propia administración la que nos adjudique el rol de vigilante/inspector y que a veces el resto de colectivos sanitarios lo perciba como una falta de capacitación para realizar las funciones anteriormente mencionadas.

El desarrollo e implantación de la historia clínica informatizada, en prácticamente todos los servicios sanitarios, nos ofrece una oportunidad única para desarrollar esta orientación clínica en la que muchos de nosotros creemos, la cual representa una meta profesional indiscutible. Reorientar los indicadores actuales de gestión, basados en datos de facturación de recetas médicas y que pivotan sobre el prescriptor, hacia indicadores de indicación-prescripción y de seguridad e idoneidad del medicamento, centrados en el paciente, es el futuro de las actividades a realizar por los FAP. Así lo están empezando a entender los Servicios de Salud, y en este sentido el Servicio de Salud de Castilla La Mancha (SESCAM) introduce para este año un objetivo en el contrato de Gestión sobre monitorización de la adecuación de la prescripción a indicaciones terapéuticas científicamente aceptadas.

Este año la SEFAP organizó su congreso anual en Toledo, bajo el lema "Seguridad del medicamento, seguridad del paciente", con el objetivo de impulsar el doble papel del farmacéutico de AP como transmisor de la cultura de la seguridad y la prevención de errores de medicación, y también como promotor de la farmacovigilancia. Los resultados del estudio sobre la seguridad de los pacientes en AP (APEAS) han puesto de manifiesto los riesgos de la asistencia sanitaria y la elevada frecuencia de aparición de acontecimientos adversos relacionados con los medicamentos $(22,4 \%)$ en la atención primaria de salud. Teniendo en cuenta el carácter evitable de los eventos adversos, la prevención de los mismos en atención primaria se perfila, por tanto, como una estrategia prioritaria en lo que atañe a la seguridad del paciente, y en este sentido el farmacéutico de atención primaria tiene aún un camino largo por recorrer, tanto en la sensibilización y formación de los profesionales sanitarios para que se reconozca el problema como en la creación de sistemas locales de notificación y en el establecimiento de prácticas de seguridad efectivas.

Y por último, esa gran asignatura pendiente para todos los colectivos sanitarios que trabajan en AP, la investigación. Se habla mucho de potenciar la investigación clí- 
nica en dicho ámbito de atención, pero ésta sigue siendo menos numerosa que en atención especializada, a pesar de que muchos de los medicamentos estudiados van a ser utilizados principalmente en AP. Los FAP no pueden perder la oportunidad de participar, junto con el resto de profesionales sanitarios, en proyectos de investigación. No se puede seguir en la inercia de que investiguen otros, pues tenemos formación suficiente y mucho que aportar.

Siempre el futuro es incierto, pero nuestro colectivo tiene que ser capaz de sortear los obstáculos, vencer el miedo y equilibrar, de un lado, el conformismo con nuestra situación actual y, de otro, la impaciencia en lanzarnos a nuevos retos, no olvidando lo hecho y conseguido hasta ahora.

\section{BIBLIOGRAFÍA}

- Ley 29/2006 de 26 de julio, de garantías y uso racional de los medicamentos y productos sanitarios (BOE $n^{\circ} 178$, de 27 de julio de 2006). Disponible en: http://www.boe.es/boe/dias/2006/07/27/ pdfs/A28122-28165.pdf.

- Galeote M, Martínez J, Beltrán C, Gangoso A, García M, Jaio N et al. Encuesta de la SEFAP sobre la Farmacia de atención primaria y la Ley 29/2006 de garantías y uso racional del medicamento y productos sanitarios. Farm Aten Prim 2008; 6(2):27-46.

- Cervera P. El Farmacéutico de Atención Primaria y el acceso a la historia clínica. Farm Aten Prim 2007; 5(3):81-82.

- Ausejo M. El modelo organizativo de la Atención Primaria: contexto profesional y científico. Farm Aten Prim 2007; 5(3):79-80.

- Estudio APEAS. Estudio sobre la seguridad de los pacientes en Atención primaria de salud. Madrid: Ministerio de Sanidad y Consumo; 2008. Disponible en: http://www.msc.es/organizacion/ sns/planCalidadSNS/docs/Estudio_APEAS_resumen.pdf .

- Garjón J. Reflexiones sobre los farmacéuticos de atención primaria ¿Cómo hemos llegado hasta aquí? Farm Aten Prim 2007; $5(1): 29$. 